OPEN ACCESS

Edited by:

Jie Yin,

Institute of Subtropical Agriculture

(CAS), China

Reviewed by:

Wutai Guan,

South China Agricultural University,

China

Guiping Guan,

Hunan Agricultural University, China

*Correspondence:

Shengyi You

shengyiyou019@163.com

Specialty section:

This article was submitted to

Gastrointestinal Sciences,

a section of the journal

Frontiers in Physiology

Received: 24 January 2019

Accepted: 11 March 2019

Published: 27 March 2019

Citation:

Zhang P, Jiao H, Wang C, Lin Y and You S (2019) Chlorogenic Acid Ameliorates Colitis and Alters Colonic Microbiota in a Mouse Model

of Dextran Sulfate Sodium-Induced

Colitis. Front. Physiol. 10:325

doi: 10.3389/fphys.2019.00325

\section{Chlorogenic Acid Ameliorates Colitis and Alters Colonic Microbiota in a Mouse Model of Dextran Sulfate Sodium-Induced Colitis}

\author{
Peng Zhang, Huanli Jiao, Chunli Wang, Yuanbang Lin and Shengyi You* \\ Department of General Surgery, Tianjin Medical University General Hospital, Tianjin, China
}

This study evaluated the mitigating effects of dietary chlorogenic acid (CGA) on colon damage and the bacterial profile in a mouse model of dextran sulfate sodium (DSS)induced colitis. C57BL/6J mice were randomly assigned to receive one of the following treatments: (i) basal diet; (ii) basal diet with 2\% CGA; (iii) basal diet with 2.5\% DSS or (iv) basal diet with 2\% CGA and 2.5\% DSS. Following a 2-week pre-treatment period, mice in the DSS and CGA-DSS groups received 2.5\% DSS in drinking water for 5 days, while the other two groups received sterile water. Compared to DSS alone, CGA was found to reduce the disease activity index, myeloperoxidase activity and tumor necrosis factor- $\alpha$ levels in colon tissues $(P<0.05)$. CGA also ameliorated DSS-induced inflammatory responses, reduced colon shortening and decreased the histological scores $(P<0.05)$. In an evaluation of the relative abundances of bacteria in the fecal microbiota, we found that CGA reversed the decrease in diversity caused by DSS and improved the relative abundance of organisms in the genus Lactobacillus $(P<0.05)$. These results indicate that CGA maintains intestinal health and reduces DSS-induced colon injury by decreasing the production of pro-inflammatory cytokines and restoring intestinal microbial diversity.

Keywords: chlorogenic acid, colitis, MPO, TNF- $\alpha$, microbiota

\section{INTRODUCTION}

Inflammatory bowel disease (IBD) involves a complex and chronic inflammatory process. Although the evidence increasingly suggests that IBD results from the exposure of a genetically susceptible host to a combination of environmental factors, the exact aetiology and pathogenesis remain unclear (Tibble et al., 2000; Zhang et al., 2014). The existing evidence indicates that IBD is caused by an abnormal mucosal immune response to intestinal microorganisms and the inappropriate secretion of cytokines in the mucosa (Cario and Podolsky, 2000; Weichselbaum and Klein, 2018). Therefore, IBD treatment primarily aims to reduce recurrent inflammation and achieve a prolonged remission (Levesque et al., 2015; Sgambato et al., 2017). Traditionally, both treatment targets have been determined by clinical symptoms rather than objective evidence of inflammatory activity. However, IBD symptoms are often a direct consequence of the inflammatory process and may differ depending on the location of inflammation (Tegtmeyer et al., 2017). 
Bioactive polyphenols, which have anti-oxidant and antiinflammatory effects and can regulate cellular signaling, could potentially be used as adjuvants to the treatment of metabolic syndrome (Santino et al., 2017). Chlorogenic acid (CGA) is an important bioactive dietary phenolic substance found widely in coffee, fruits and vegetables (Naveed et al., 2018). Studies have shown that CGA has anti-oxidant, anti-bacterial and antiinflammatory effects (Zhao et al., 2008; Kamiyama et al., 2015; Tsang et al., 2016; Zheng et al., 2016). For example, CGA inhibited the production of interleukin (IL)-8 in Caco-2 human intestinal cells in response to tumor necrosis factor (TNF) $-\alpha$ and $\mathrm{H}_{2} \mathrm{O}_{2}$, while studies in C57BL/6 mice found that CGA could alleviate dextran sulfate sodium (DSS)-induced weight loss, diarrhea, fecal blood and colon shortening. These data suggest that CGA can prevent intestinal inflammation (Shin et al., 2015). Other studies of rodent models have shown that CGA can inhibit lipopolysaccharide-induced myeloperoxidase (MPO) activity in the lung (Zatorski et al., 2015).

Colon microbes play an essential role in human health and have been associated with various diseases, including irritable bowel syndrome (Kassinen et al., 2007), autism (Li and Zhou, 2016) and obesity (Zhang et al., 2009). Most ingested CGA is not absorbed in the small intestine and reaches the colon, where it is converted into several metabolites by the local microbial community. Studies have shown that Bifidobacterium animalis can hydrolyse and alter the fate of CGA and thus affect the composition of the microbiota (Tomas-Barberan et al., 2014). Another study found that a 10 -h course of CGA treatment increased the numbers of Bifidobacterium spp. and members of the Clostridium coccoides-Eubacterium rectale group in vitro and promoted the expansion of select bacteria, compared with the control group (Mills et al., 2015). Although colitis has been very well studied, evidence regarding the role of CGA in the alleviation of this disease is scarce. In this study, the effects of CGA on colonic microbial composition and pro-inflammatory factors were explored in a rodent model of DSS-induced colitis. In vivo experiments were also conducted to investigate whether CGA could prevent intestinal inflammation.

\section{MATERIALS AND METHODS}

\section{Animals and Experimental Treatments}

For this study, all procedures involving animals were approved by the Animal Ethics Committee of General Hospital of Tianjin Medical University and conformed in all respects with the Guidelines for the Care and Use of Laboratory Animals of Tianjin Medical University. The female C57BL/6J mice utilized in this study were obtained from the SLAC Laboratory Animal Centre (Shanghai, China) at 6-7 weeks of age. Mice were housed in a pathogen-free colony in accordance with standard laboratory conditions, including a temperature of $22-24^{\circ} \mathrm{C}$, humidity of $40-$ $60 \%$ and 12-h daily light/dark cycle. After a 7-day adaptation period, 40 mice were randomly assigned to one of four discrete treatment groups: (1) basal diet $(\mathrm{CON})$; (2) basal diet with 2\% CGA (CGA); (3) basal diet with 2.5\% DSS (DSS, MW 5000, KAYON Biotechnology Co., Ltd.) and (4) basal diet with 2\% CGA and $2.5 \%$ DSS (CGA-DSS). CGA was dissolved in sterile drinking water for administration. All of the mice had access to food and water ad libitum, and the latter was changed twice weekly. During a 20-day experiment, the drinking water was supplemented or not with $2.5 \%$ DSS for the last 5 days. The body weight of each mouse was recorded at the end of the experiment.

\section{Sample Collection}

All mice were sacrificed according to standard procedures. The colon was removed from each animal and rinsed in physiological saline to remove fecal residue. The weight and length of each organ were recorded to determine the inflammation index. Next, samples from various segments of each colon were fixed in $4 \%$ buffered formaldehyde and embedded in paraffin. Fourmicrometer-thick slices of the paraffin-embedded tissues were stained with haematoxylin and eosin (H\&E) in accordance with standard procedures for the histological evaluation of colonic damage. The remaining colonic tissue samples and digests were stored in frozen liquid nitrogen for further measurements of biological parameters.

\section{Colitis Disease Activity Index (DAl)}

Tissue samples from the distal colon $(5 \mu \mathrm{m})$ were stained with $\mathrm{H} \& \mathrm{E}$ and subjected to a microscopic analysis to determine the colon histological score, as proposed by Cooper et al. (1993). The following scoring system was used (maximum score $=10$ ): 0 (rare) $=$ severe inflammatory cell infiltration; $1=$ marginally dispersed cell infiltrate; 2 = moderately increased cell infiltrate with the formation of occasional cell foci and $3=$ large areas of cell infiltration causing a severe loss of tissue architecture. Additionally, the following scoring system was used to determine the extent of injury: 0 = none; $1=$ mucosal; 2 = mucosal and submucosal, and 3 = transmural. Crypt damage was scored as follows: 0 = intact crypts; 1 = damage to the basal one-third; 2 = damage to the basal two-thirds damaged, $3=$ only surface epithelium remains intact, and $4=$ loss of entire crypt and epithelium (Backer et al., 2017).

\section{Assessment of Leukocyte Involvement}

Myeloperoxidase activity, a marker of neutrophilic infiltration, was assessed using the method described by Grisham et al. (1990) with slight modifications. A distal colon sample from each mouse was excised, immediately rinsed with ice-cold saline, blotted dry and frozen at $-70^{\circ} \mathrm{C}$. Subsequently, the tissue samples were thawed, weighed and homogenized in 10 volumes of $50 \mathrm{mM}$ phosphate-buffered saline (PBS; $\mathrm{pH}$ 7.4). The homogenates were then centrifuged at 20,000 $g$ and $4^{\circ} \mathrm{C}$ and re-homogenized in 10 volumes of $50 \mathrm{mM}$ PBS $(\mathrm{pH}$ 6.0) containing $0.5 \%$ hexadecyltrimethylammonium bromide (HETAB) and $10 \mathrm{mM}$ ethylenediamine tetra-acetic acid (EDTA). Subsequently, the homogenates were subjected to a freeze/thaw cycle and a brief period of sonication, diluted in 50 volumes of $50 \mathrm{mM}$ PBS ( $\mathrm{pH} \mathrm{6)}$ ) and added to $50 \mathrm{ml}$ of a solution containing $o$-Dianisidine dihydrochloride $(0.067 \%)$, HETAB $(0.5 \%)$ and hydrogen peroxide $(0.003 \%)$. Complete sample reaction mixtures were placed in separate wellsand incubated in darkness for 5 min. A microplate reader (Labsystem Multiskan EX, Helsinki, 
Finland) was used to measure the changes in absorbance at $450 \mathrm{~nm}$ in accordance with the user's manual. The results are expressed in units of $\mathrm{U} / \mathrm{mg}$ protein.

\section{Determination of TNF- $\alpha$ Level}

Colon samples were homogenized in ice-cold PBS as previously described and centrifuged at 3,000 $\mathrm{g}$ for $10 \mathrm{~min}$. The TNF- $\alpha$ levels in the supernatants were determined using an enzymelinked immunosorbent assay kit (Dou et al., 2013). Moreover, the degree of tissue inflammation was monitored using the tissue TNF- $\alpha$ activity level, which exhibits a linear relationship with neutrophilic infiltration in inflamed tissues. The TNF- $\alpha$ activity was determined in colonic samples adjacent to the installation point with a kits in accordance with the manufacturer's instructions (CytoStore, Calgary, AB, Canada). The results from each sample are expressed in units of $\mathrm{pg} / \mathrm{mg}$ of protein.

\section{S rRNA Sequencing}

Thawed samples of colonic contents were mixed with PBS and homogenized using a high-speed homogenizer. The QIAamp DNA Stool Mini Kit was used to extract the total bacterial genome from each sample according to the manufacturer's protocol (Qiagen, Hilden, Germany). A polymerase chain reaction (PCR) assay containing the Phusion ${ }^{\circledR}$ High-Fidelity PCR Master Mix and GC Buffer kit (New England Biolabs, Ipswich, MA, United States) and the primers 341F (5'-CCTAYGGGRBGCASCAG-3') and 806R (5'GGACTACNNGGGTATCTAAT- $3^{\prime}$ ) was used to amplify the $16 \mathrm{~S}$ rRNA (V3-V4 region) genes in each sample. The following PCR conditions were applied: initial denaturation at $98^{\circ} \mathrm{C}$ for $30 \mathrm{~s}$; 30 cycles of $98^{\circ} \mathrm{C}$ for $10 \mathrm{~s}, 55^{\circ} \mathrm{C}$ for $30 \mathrm{~s}$ and $72^{\circ} \mathrm{C}$ for $30 \mathrm{~s}$ and a final extension at $72^{\circ} \mathrm{C}$ for $5 \mathrm{~min}$. The resulting PCR products were stored at $4^{\circ} \mathrm{C}$ until required. Later, the target fragments were detected by subjecting the PCR products to $2 \%$ agarose gel electrophoresis and were collected using the QIAquick Gel Extraction Kit (Qiagen, Germantown, MD, United States). A gene library was constructed using the Illumina TruSeq ${ }^{\circledR}$ DNA PCR-Free Sample Preparation Kit (Illumina Inc., San Diego, CA, United States), quantified using Qubit and real-time PCR and sequenced on a HiSeq2500 PE250 device (Illumina Inc.).

\section{Statistical Analysis}

SPSS, version 20 (IBM Corp., Armonk, NY, United States) was used to conduct the statistical analysis. A one-way analysis of variance with Duncan's multiple range test was used to determine significant differences between the groups. A $P$-value of $<0.05$ was considered to indicate a significant difference.

\section{RESULTS}

\section{CGA Ameliorates DSS-Induced Colitis}

According to the initial random group allocation, mice in the CGA and CGA-DSS groups were treated with CGA for 14 days. The other groups received the basal diet for the same length of time. Mice in the DSS and CGA-DSS groups then received $2.5 \%$ DSS for the last 5 days of the study period, after which the final body weight and DAI were recorded (Figure 1). The results demonstrate that in the CGA-DSS group dietary CGA significantly reduced the DAI, compared to the DSS group $(P<0.05)$, and alleviated DSS-induced colitis. Despite CGA failing to reverse the DSS-induced weight loss, compared with mice in the DSS group, the mice in the CGA group gained weight $(P<0.05)$.

\section{The Effects of CGA on MPO Activity and TNF- $\alpha$ Levels in Mice With DSS-Induced Colitis}

Myeloperoxidase is a potential marker of tissue inflammation, tissue injury and neutrophil infiltration (Krawisz et al., 1984). The experimental results indicate increased MPO activity in the DSS group relative to the other groups $(P<0.05)$, as shown in Figure 2A. TNF- $\alpha$ also appears to play a significant role in the inflammatory process associated with ulcerative colitis (Moldoveanu et al., 2015). In this study, mice exposed to DSS exhibited a significant increase in TNF- $\alpha$ levels in the colon, whereas CGA treatment significantly mitigated this response $(P<0.05)$ as shown in Figure 2B. These data suggest that CGA exerts an anti-inflammatory effect by reducing neutrophil infiltration and pro-inflammatory cytokine production in the colon.
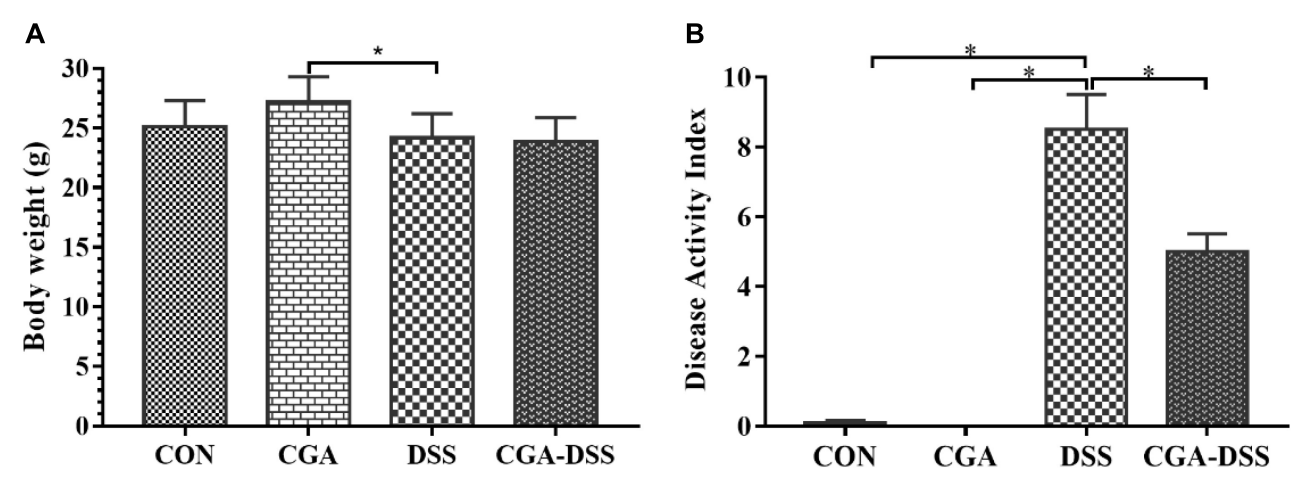

FIGURE 1 | Effects of chlorogenic acid (CGA) on dextran sulfate sodium (DSS)-induced colitis. (A) The body weights of mice treated with CGA. (B) The disease activity indices (DAI) of mice after a 5-day treatment with $2.5 \%$ DSS. *indicates a $P$-value $<0.05$, compared with DSS. 

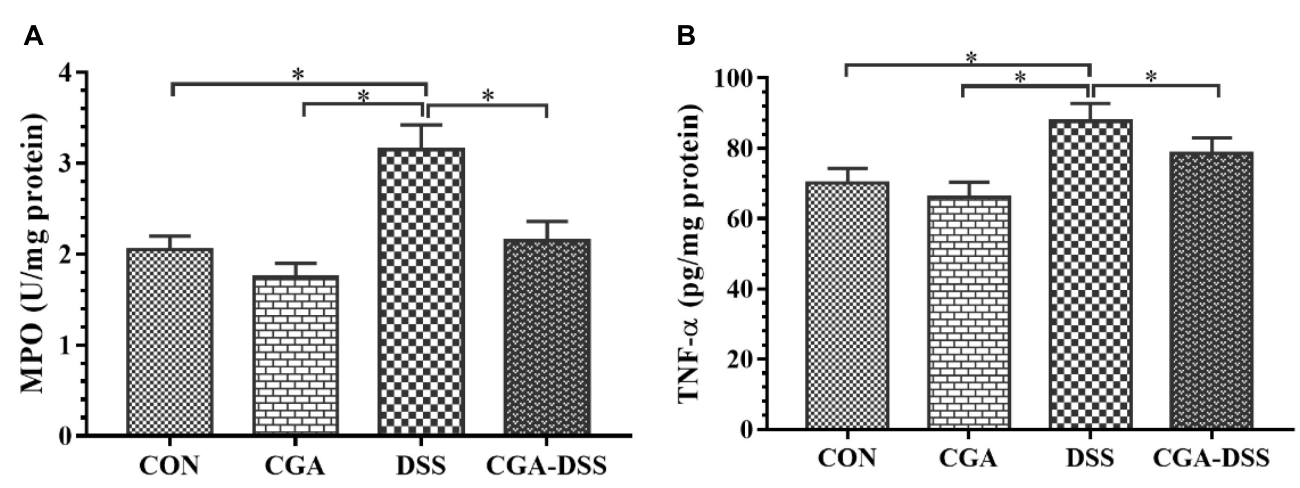

FIGURE 2 | Effects of chlorogenic acid (CGA) on (A) myeloperoxidase (MPO) activity and (B) tumor necrosis factor (TNF)- $\alpha$ levels in a mouse model of dextran sulfate sodium (DSS)-induced colitis. *indicates a $P$-value $<0.05$.

A CON

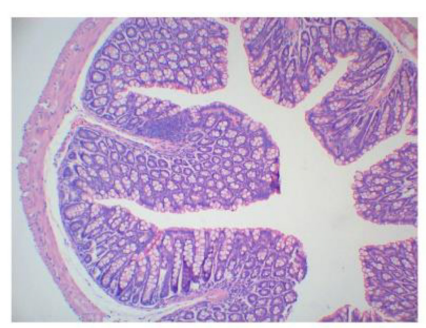

C DSS

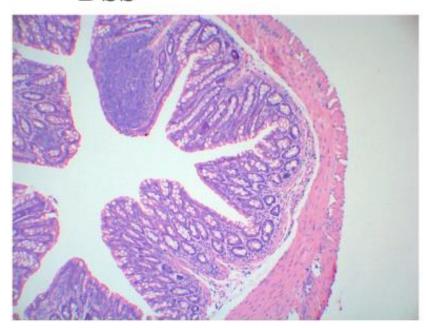

B CGA

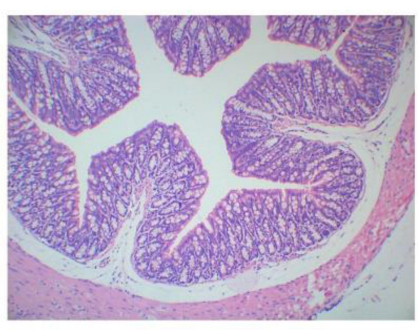

D CGA+DSS

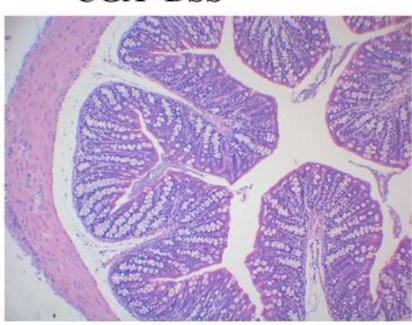

E

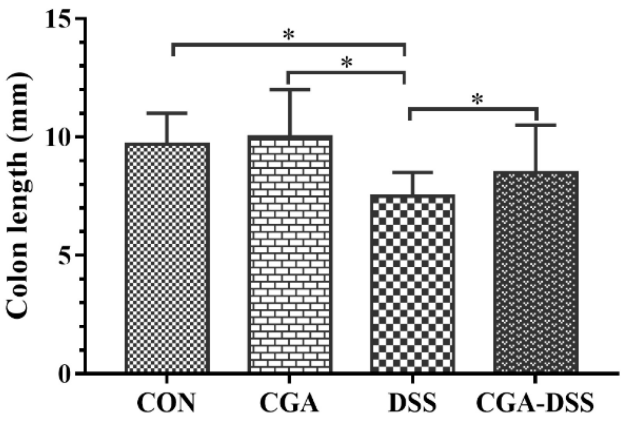

$\mathbf{F}$

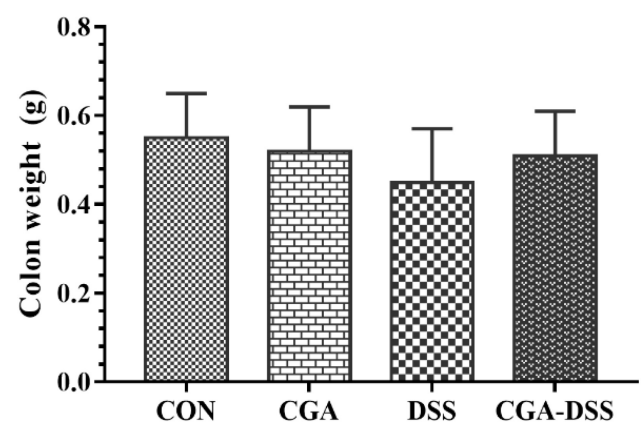

G

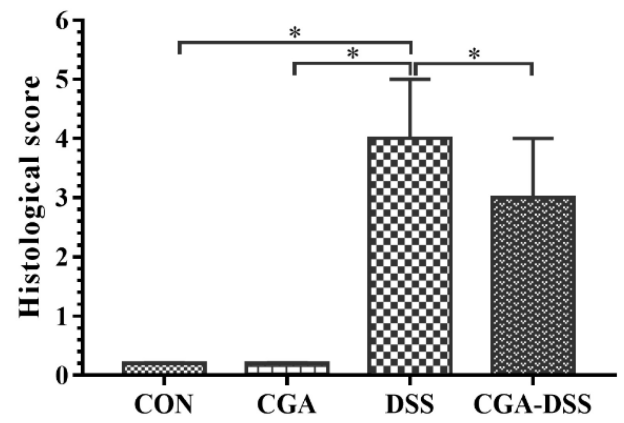

FIGURE 3 | Colon tissue damage in response to dextran sulfate sodium (DSS) treatment. Effects of chlorogenic acid (CGA) on colon histology [A: control (CON) group, B: CGA group, C: DSS group and D: CGA-DSS group], colon weight (E), colon length (F), and histological score (G). Original magnification: 100x. *indicates a $P$-value $<0.05$. 


\section{The Effects of CGA on Macroscopic and Histologic Observations in DSS-Induced Colitis}

A histological comparison of colonic tissues from the control and DSS groups revealed multiple erosive lesions and an extensive infiltration of inflammatory cells that predominantly comprised macrophages, lymphocytes and neutrophils, along with irregular eosinophils, in the latter (Figures $\mathbf{3 A - D}$ ). The levels of inflammatory cell infiltration and observable degree of tissue damage in the colonic tissues were assessed using the previously described histological scoring system. After a 5-day period of exposure to DSS in drinking water, mice in the DSS group exhibited an advanced inflammatory response. However, CGA had a mitigating effect on DSS-induced inflammatory markers (Figure 3G). Colon shortening is an indicator of colitis and inflammation (Siegmund, 2002). Notably, the DSS group exhibited significant colon shortening, compared to the other groups (Figure 3E, $P<0.05$ ). However, no significant intergroup differences in colon weight were observed (Figure 3F).

\section{The Effect of CGA on Diversity in the Colonic Microbiota}

Next, the V3-V4 regions of 16S rRNA isolated from the colonic digest samples were sequenced. The Shannon
(Figure 4A), Simpson (Figure 4B), Chao (Figure 4C) and ACE indices (Figure 4D) were used to measure colonic microbial diversity. Treatment with DSS led to decreases in both the Shannon $(P=0.042)$ and Simpson indices $(P=0.035)$, whereas CGA treatment reversed these changes. By contrast, no significant differences were observed in the Chao and ACE indices.

\section{The Effect of CGA on Microbial Abundance at the Phylum Level}

The major bacterial phyla in the colonic digest samples included Bacteroidetes, Firmicutes, Proteobacteria, and Verrucomicrobia, which accounted for approximately $90 \%$ of the microbiota. Bacteroidetes was most prevalent, accounting for $60.06,49.72,29.85$, and $37.29 \%$ of the colonies in the CON, CGA, DSS, and CGA-DSS groups, respectively. The corresponding proportions of Firmicutes were $27.14,29.45,33.78$, and $36.95 \%$, respectively, while those of Proteobacteria were 8.62, 10.00, 8.37, and 8.20\%, respectively (Figure 5A). DSS treatment decreased the relative abundance of Bacteroidetes (Figure 5B, $P<0.05$ ), and this change was not reversed by CGA treatment. DSS treatment also decreased the relative abundance of Verrucomicrobia (Figure 5C).

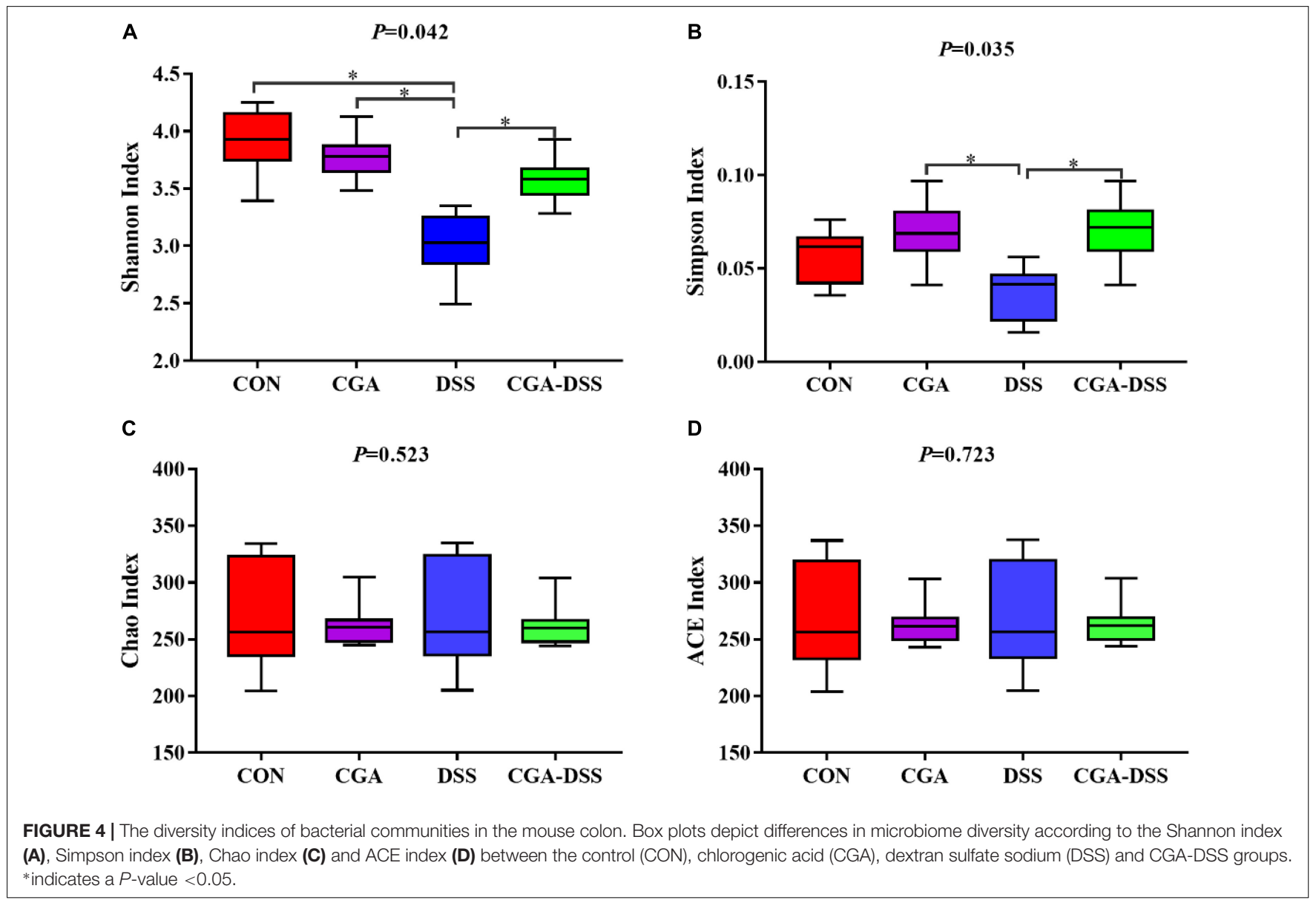


A

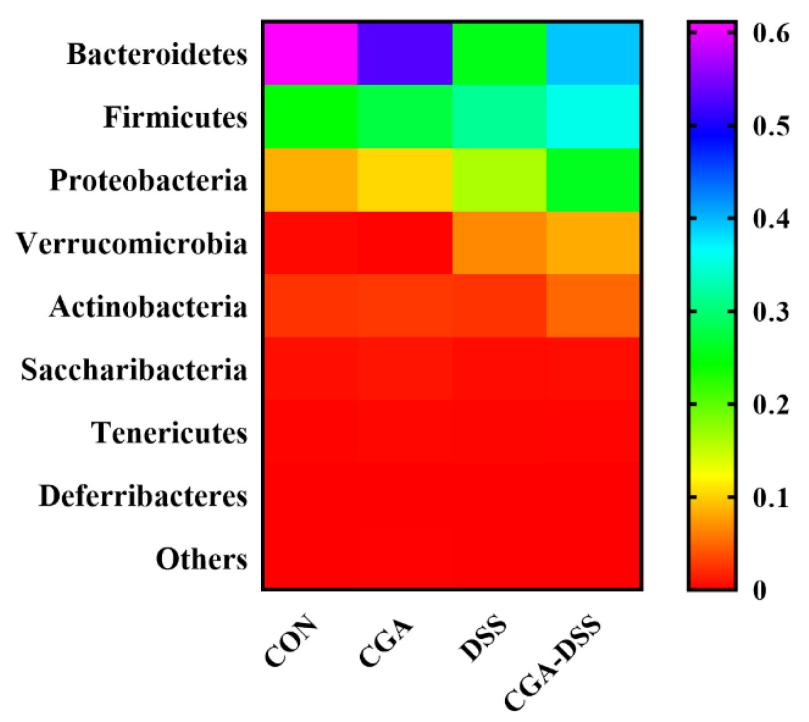

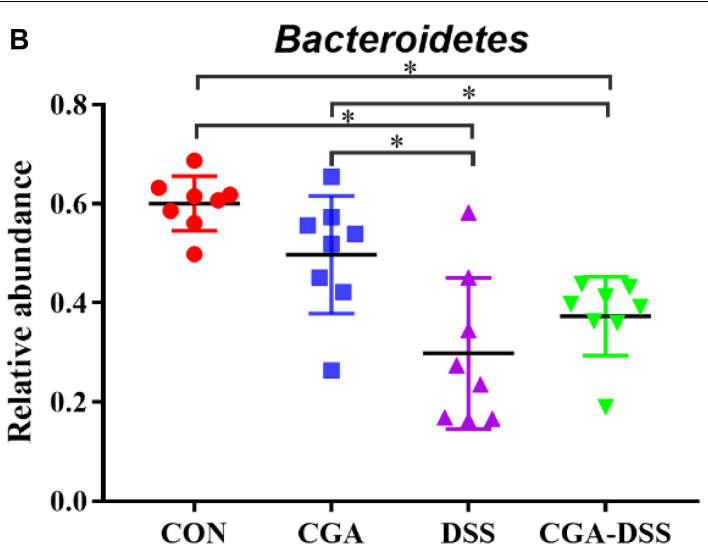

c Verrucomicrobia

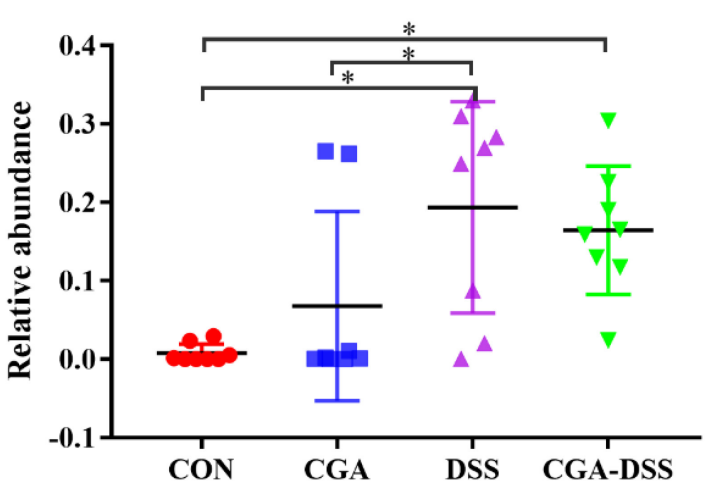

FIGURE 5 | Analysis of the colon microbial composition at the phylum level. (A) Relative abundances of microbial phyla in the mouse colons. The relative abundances of Bacteroidetes (B) and Verrucomicrobia (C) were compared between the control (CON), chlorogenic acid (CGA), dextran sulfate sodium (DSS), and CGA-DSS groups. *indicates a $P$-value $<0.05$.

\section{The Effect of CGA on Microbial Abundance at the Genus Level}

The 10 most abundant microbial genera are shown in Figure 6. Bacteroides (14.86), Parasutterella (2.72\%), and Helicobacter (3.44\%) were predominant in the CON group, whereas Akkermansia, Bacteroides and Lactobacillus were the three major strains in the CGA, DSS, and CGA-DSS groups. In the latter groups, the proportions of Bacteroides were 12.56, 14.05, and $9.44 \%$, respectively, while those of Lactobacillus and Akkermansia were $6.58,9.39$, and $12.47 \%$, respectively and $6.27,15.94$, and $18.87 \%$, respectively. The proportion of Akkermansia increased in the CGA-DSS group relative to the CON and CGA groups and in the DSS group relative to the CON group. CGA also increased the relative abundance of Lactobacillus.

\section{DISCUSSION}

Continuous exposure to external stressors, such as foods, bacteria and environmental chemicals, induces a certain level of intestinal inflammation, although intestinal tissue damage and dysfunction may also play a role (Shimizu, 2017). CGA, an esterification product of caffeic acid and quinic acid, is one of the most abundant polyphenols in the human diet and has been shown to inhibit inflammatory responses in intestinal cells (Zhang et al., 2010). In this study, treatment with 2\% CGA was shown to prevent weight loss and reduce the DAI. Furthermore, CGA reduced MPO activity and TNF- $\alpha$ levels in IBD-affected colons and ameliorated DSS-induced colon shortening and inflammatory responses. Moreover, CGA reduced the negative effects of DSS on intestinal microbial diversity and increased the relative abundance of Lactobacillus spp.

Intestinal dysfunction may affect the absorption of nutrients and, consequently, body weight. Furthermore, colon shortening is a sign and symptom of inflammation (Gearry et al., 2009). Studies of wild-type mice have shown that DSS-induced colitis significantly reduces body weight gain, increases epithelial permeability, rectal bleeding, colon length shortening, ulcer formation, inflammatory cell infiltration and goblet cell loss (Gadaleta et al., 2011). In a BALB/c mouse model of DSS-induced colitis, green tea polyphenols appeared to attenuate colitis by reducing the levels of TNF- $\alpha$ and serum amyloid A. Notably, the effects of green tea polyphenols on colitis were similar to those of sulfasalazine (Oz et al., 2013). Another study found that polyphenols from extra virgin olive oil could reduce the DAI and decrease the expression of monocyte chemoattractant protein 


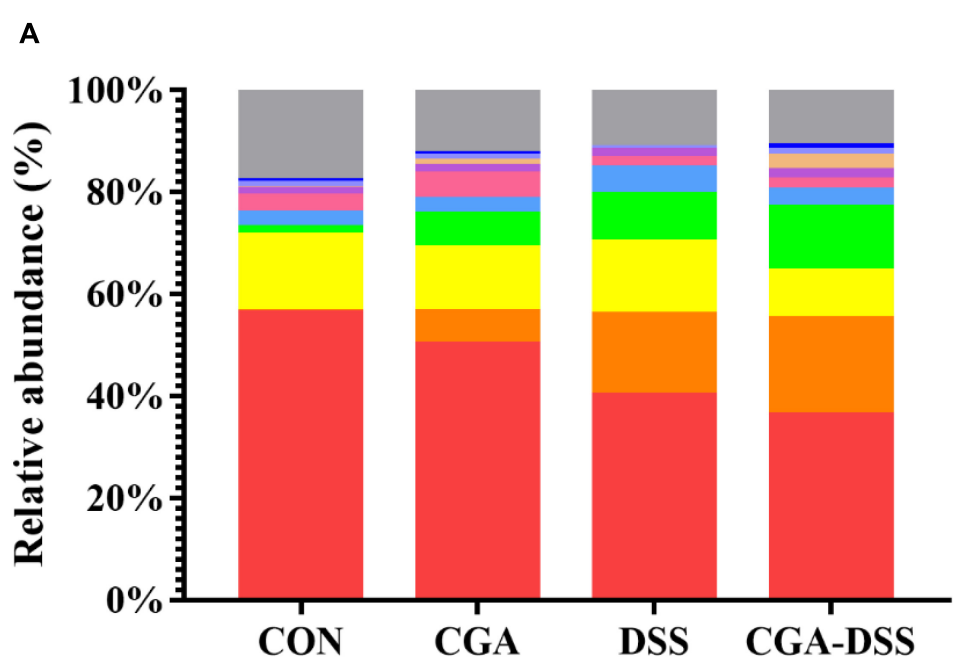

Others
Psychrobacter
Corynebacterium
Facklamia
Desulfovibrio
Helicobacter
Parasutterella
Lactobacillus
Bacteroides
Akkermansia
unclassified

B

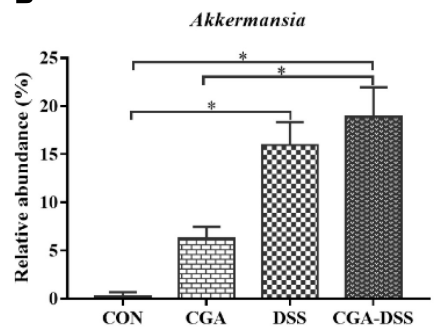

E

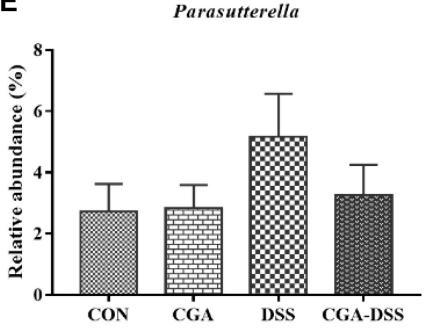

C

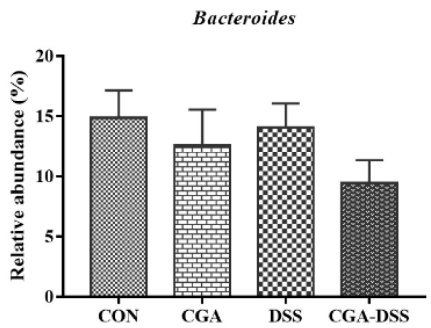

$\mathbf{F}$

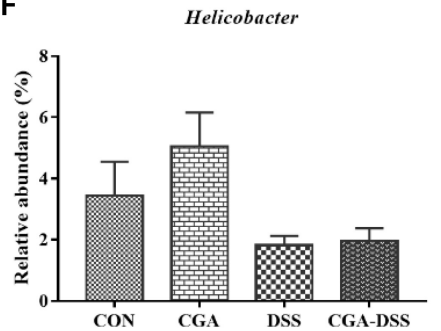

D

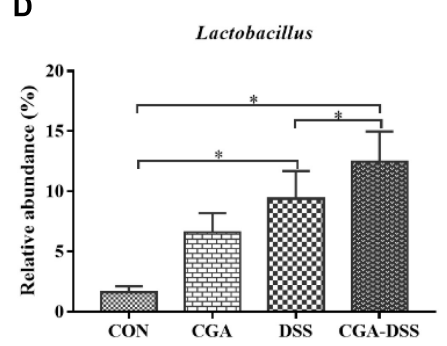

$\mathbf{G}$

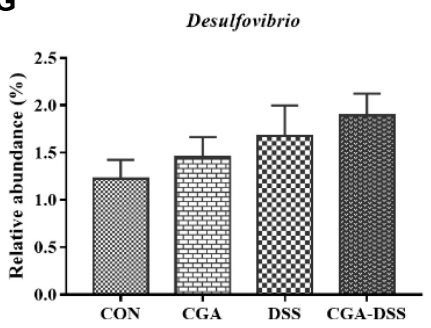

FIGURE 6 | Analysis of the colon microbial composition at the genus level. (A) Relative abundances of microbial genera in the mouse colons. The relative abundances of Akkermansia (B), Bacteroides (C), Lactobacillus (D), Parasutterella (E), Helicobacter (F) and Desulfovibrio (G) spp. were compared between the control (CON), chlorogenic acid (CGA), dextran sulfate sodium (DSS), and CGA-DSS groups. *indicates a $P$-value $<0.05$.

(MCP)-1, TNF- $\alpha$, cyclooxygenase (COX)-2 and inducible nitric oxide synthase (iNOS) in colon tissues when compared with DSS treatment alone. These findings suggest that dietary polyphenols may be beneficial for the treatment of ulcerative colitis (SanchezFidalgo et al., 2013). Our study revealed that the addition of CGA to drinking water reduced the DAI and the severity of colon damage and shortening in response to DSS.

Cytokines are characteristic factors of IBD, wherein they act as key pathophysiological regulators of the occurrence, development and final resolution of inflammation (Strober and Fuss, 2011). In the past few decades, studies of cytokines in the context of IBD and other mucosal inflammatory conditions have been fruitful. These studies not only have provided us with important insights into the mechanisms of these diseases, but have also indicated directions for new treatment methods. Interactions between TNF family receptors and their ligands play important roles in the formation of key immune responses, including programmed cell death and lymphocyte co-stimulation (Meylan et al., 2011). CGA can inhibit the production of TNF, IL6 , INF- $\gamma$, MCP-1 and macrophage inflammatory protein-l $\alpha$ by human peripheral blood monocytes in response to pathogenic bacteria (Krakauer, 2002). Ellagic acid was shown to reduce the expression of NF- $\kappa$ B, COX-2, iNOS, TNF- $\alpha$, and IL- 6 in 1, 2-dimethylhydrazine-induced colon cancer (Umesalma and Sudhandiran, 2010). MPO, a primarily a neutrophilic enzyme, is used as a quantitative indicator of inflammation because of the correlation between MPO activity and the histological detection of neutrophilic infiltration in the colon (Cetinkaya et al., 2006; 
Dost et al., 2009; Guan and Lan, 2018). In a previous rodent model study, $50 \mathrm{mg} / \mathrm{kg}$ CGA inhibited an increase in MPO activity in response to LPS and suppressed the migration of polymorphonuclear neutrophils to the lungs, as detected in bronchoalveolar lavage fluid. CGA also significantly reduced iNOS activity in lung tissues and thus inhibited the release of nitric oxide by LPS (Zhang et al., 2010). Another study found that treatment with $50 \mathrm{mg} / \mathrm{kg}$ CGA reduced the levels of MPO in gastric tissues, while a morphological analysis revealed the inhibition of neutrophilic infiltration into damaged tissues (Shimoyama et al., 2013). In this study, 2\% CGA was shown to reduce MPO activity and DSS-induced damage in colon tissues.

Studies of animal models of IBD have suggested that intestinal inflammation relies heavily on a triggering event mediated by the intestinal microflora. Changes in the intestinal microflora can significantly affect both host immunity and mucosal inflammation (Hooper and Gordon, 2001; Ott et al., 2004; Huang et al., 2018; Li et al., 2018). Some changes in the microbial community are consistently observed in the context of intestinal inflammation, including reduced diversity (especially Firmicutes) and the presence of uncommon bacteria and increased concentrations of Escherichia coli (including pathogenic strains) (Sokol et al., 2006, 2008; Frank et al., 2007). Studies have shown that the oral administration of DSS can reduce the abundance of Akkermansia muciniphila and Bacteroides acidifaciens in feces (Kang et al., 2013; Yin et al., 2018). Recent studies of an IL10 ${ }^{-/-}$mouse model of IBD have identified NLRP6 as an important inhibitor of spontaneous colitis, whereas a lack of NLRP6 leads to the enrichment of Akkermansia muciniphila (Seregin et al., 2017; Zhang et al., 2018). Further studies of mouse models of colitis found that Lactobacillus paracasei could reduce intestinal inflammation and the expression of pro-inflammatory factors in the mucosa (Oliveira et al., 2011; Wu and Tian, 2017; Azad et al., 2018). Treatment with a polyphenol-rich cranberry extract was shown to significantly increase the proportion of mucus-degrading Akkermansia spp. in a metagenomic sample (Anhe et al., 2015). Moreover, a phenolic extract of grape pomace/grape cider (1 $\mathrm{mg} / \mathrm{mL}$ ) significantly increased the Lactobacillus acidophilus biomass in liquid medium, while exposure to the highest concentration of phenolic compounds $(5000 \mathrm{~g} / \mathrm{disk})$ had no

\section{REFERENCES}

Anhe, F. F., Roy, D., Pilon, G., Dudonne, S., Matamoros, S., Varin, T. V., et al. (2015). A polyphenol-rich cranberry extract protects from diet-induced obesity, insulin resistance and intestinal inflammation in association with increased Akkermansia spp. population in the gut microbiota of mice. Gut 64, 872-883. doi: 10.1136/gutjnl-2014-307142

Azad, M. A. K., Sarker, M., Li, T., and Yin, J. (2018). Probiotic species in the modulation of gut microbiota: an overview. Biomed. Res. Int. 2018:9478630. doi: 10.1155/2018/9478630

Backer, V., Cheung, F. Y., Siveke, J. T., Fandrey, J., and Winning, S. (2017). Knockdown of myeloid cell hypoxia-inducible factor-1alpha ameliorates the acute pathology in DSS-induced colitis. PLoS One 12:e0190074. doi: 10.1371/ journal.pone.0190074

Cario, E., and Podolsky, D. K. (2000). Differential alteration in intestinal epithelial cell expression of toll-like receptor 3 (TLR3) and TLR4 in inflammatory inhibitory effect on the growth of Lactobacillus acidophilus in an agar diffusion assay (Hervert-Hernandez et al., 2009). This study demonstrated that 2\% CGA could improve microbial diversity in the colon, particularly the relative abundances of Akkermansia and Lactobacillus, and suggests that CGA could potentially restore the microecological disorder induced by DSS.

\section{CONCLUSION}

In conclusion, our data provide insights into the role of CGA as a regulator of immunity and microbial diversity in the colon. Although these experiments were conducted in a mouse model of DSS-induced colitis, CGA could similarly inhibit the release of proinflammatory cytokines, such as MPO and TNF- $\alpha$, in ulcerative colitis. Moreover, CGA was shown to have positive effects on the intestinal microbiota. Specifically, this polyphenol led to increases in microbial diversity and the relative abundances of Akkermansia and Lactobacillus. Our study thus demonstrates the possible use of CGA as a therapeutic adjunct to colitis treatment.

\section{DATA AVAILABILITY}

All datasets generated for this study are included in the manuscript and/or the supplementary files.

\section{AUTHOR CONTRIBUTIONS}

PZ, HJ, CW, and YL finished all the experiments. PZ and HJ performed the statistical analysis. PZ finished the first draft of the manuscript. SY critically revised the manuscript. All the authors read and approved the manuscript.

\section{FUNDING}

This Project was supported by Science and Technology Development Fund Project of Tianjin no. 20130138.

bowel disease. Infect. Immun. 68, 7010-7017. doi: 10.1128/IAI.68.12.7010-7017. 2000

Cetinkaya, A., Bulbuloglu, E., Kantarceken, B., Ciralik, H., Kurutas, E. B., Buyukbese, M. A., et al. (2006). Effects of L-carnitine on oxidant/antioxidant status in acetic acid-induced colitis. Dig. Dis. Sci. 51, 488-494. doi: 10.1007/ s10620-006-3160-9

Cooper, H. S., Murthy, S. N., Shah, R. S., and Sedergran, D. J. (1993). Clinicopathologic study of dextran sulfate sodium experimental murine colitis. Lab. Invest. 69, 238-249.

Dost, T., Ozkayran, H., Gokalp, F., Yenisey, C., and Birincioglu, M. (2009). The effect of Hypericum perforatum (St. John's Wort) on experimental colitis in rat. Dig. Dis. Sci. 54, 1214-1221. doi: 10.1007/s10620-008-0477-6

Dou, W., Zhang, J., Zhang, E., Sun, A., Ding, L., Chou, G., et al. (2013). Chrysin ameliorates chemically induced colitis in the mouse through modulation of a PXR/NF-kappaB signaling pathway. J. Pharmacol. Exp. Ther. 345, 473-482. doi: 10.1124 /jpet.112.201863 
Frank, D. N., St Amand, A. L., Feldman, R. A., Boedeker, E. C., Harpaz, N., and Pace, N. R. (2007). Molecular-phylogenetic characterization of microbial community imbalances in human inflammatory bowel diseases. Proc. Natl. Acad. Sci. U.S.A. 104, 13780-13785. doi: 10.1073/pnas.0706625104

Gadaleta, R. M., van Erpecum, K. J., Oldenburg, B., Willemsen, E. C., Renooij, W., Murzilli, S., et al. (2011). Farnesoid X receptor activation inhibits inflammation and preserves the intestinal barrier in inflammatory bowel disease. Gut 60, 463-472. doi: 10.1136/gut.2010.212159

Gearry, R. B., Irving, P. M., Barrett, J. S., Nathan, D. M., Shepherd, S. J., and Gibson, P. R. (2009). Reduction of dietary poorly absorbed short-chain carbohydrates (FODMAPs) improves abdominal symptoms in patients with inflammatory bowel disease-a pilot study. J. Crohn's Colitis 3, 8-14. doi: 10.1016/j.crohns.2008. 09.004

Grisham, M. B., Benoit, J. N., and Granger, D. N. (1990). Assessment of leukocyte involvement during ischemia and reperfusion of intestine. Methods Enzymol. 186, 729-742. doi: 10.1016/0076-6879(90)86172-R

Guan, G., and Lan, S. (2018). Implications of antioxidant systems in inflammatory bowel disease. Biomed. Res. Int. 2018:1290179. doi: 10.1155/2018/1290179

Hervert-Hernandez, D., Pintado, C., Rotger, R., and Goni, I. (2009). Stimulatory role of grape pomace polyphenols on Lactobacillus acidophilus growth. Int. J. Food Microbiol. 136, 119-122. doi: 10.1016/j.ijfoodmicro.2009.09.016

Hooper, L. V., and Gordon, J. I. (2001). Commensal host-bacterial relationships in the gut. Science 292, 1115-1118. doi: 10.1126/science.1058709

Huang, L., Gao, R., Yu, N., Zhu, Y., Ding, Y., and Qin, H. (2018). Dysbiosis of gut microbiota was closely associated with psoriasis. Sci. China Life Sci. doi: 10.1007/s11427-018-9376-6 [Epub ahead of print].

Kamiyama, M., Moon, J. K., Jang, H. W., and Shibamoto, T. (2015). Role of degradation products of chlorogenic acid in the antioxidant activity of roasted coffee. J. Agric. Food Chem. 63, 1996-2005. doi: 10.1021/jf5060563

Kang, C. S., Ban, M., Choi, E. J., Moon, H. G., Jeon, J. S., Kim, D. K., et al. (2013). Extracellular vesicles derived from gut microbiota, especially Akkermansia muciniphila, protect the progression of dextran sulfate sodium-induced colitis. PLoS One 8:e76520. doi: 10.1371/journal.pone.0076520

Kassinen, A., Krogius-Kurikka, L., Makivuokko, H., Rinttila, T., Paulin, L., Corander, J., et al. (2007). The fecal microbiota of irritable bowel syndrome patients differs significantly from that of healthy subjects. Gastroenterology 133 , 24-33. doi: 10.1053/j.gastro.2007.04.005

Krakauer, T. (2002). The polyphenol chlorogenic acid inhibits staphylococcal exotoxin-induced inflammatory cytokines and chemokines. Immunopharmacol. Immunotoxicol. 24, 113-119. doi: 10.1081/IPH-120003407

Krawisz, J. E., Sharon, P., and Stenson, W. F. (1984). Quantitative assay for acute intestinal inflammation based on myeloperoxidase activity. assessment of inflammation in rat and hamster models. Gastroenterology 87, 1344-1350.

Levesque, B. G., Sandborn, W. J., Ruel, J., Feagan, B. G., Sands, B. E., and Colombel, J. F. (2015). Converging goals of treatment of inflammatory bowel disease from clinical trials and practice. Gastroenterology 148, 37.e1-51.e1. doi: 10.1053/j. gastro.2014.08.003

Li, M., Wu, Y., Hu, Y., Zhao, L., and Zhang, C. (2018). Initial gut microbiota structure affects sensitivity to DSS-induced colitis in a mouse model. Sci. China Life Sci. 61, 762-769. doi: 10.1007/s11427-017-9097-0

Li, Q., and Zhou, J. M. (2016). The microbiota-gut-brain axis and its potential therapeutic role in autism spectrum disorder. Neuroscience 324, 131-139. doi: 10.1016/j.neuroscience.2016.03.013

Meylan, F., Song, Y. J., Fuss, I., Villarreal, S., Kahle, E., Malm, I. J., et al. (2011). The TNF-family cytokine TL1A drives IL-13-dependent small intestinal inflammation. Mucosal Immunol. 4, 172-185. doi: 10.1038/mi.2010.67

Mills, C. E., Tzounis, X., Oruna-Concha, M. J., Mottram, D. S., Gibson, G. R., and Spencer, J. P. (2015). In vitro colonic metabolism of coffee and chlorogenic acid results in selective changes in human faecal microbiota growth. Br. J. Nutr. 113, 1220-1227. doi: 10.1017/S0007114514003948

Moldoveanu, A. C., Diculescu, M., and Braticevici, C. F. (2015). Cytokines in inflammatory bowel disease. Rom. J. Intern. Med. 53, 118-127. doi: 10.1515/ rjim-2015-0016

Naveed, M., Hejazi, V., Abbas, M., Kamboh, A. A., Khan, G. J., Shumzaid, M., et al. (2018). Chlorogenic acid (CGA): A pharmacological review and call for further research. Biomed. Pharmacother. 97, 67-74. doi: 10.1016/j.biopha.2017.10.064

Oliveira, M., Bosco, N., Perruisseau, G., Nicolas, J., Segura-Roggero, I., Duboux, S., et al. (2011). Lactobacillus paracasei reduces intestinal inflammation in adoptive transfer mouse model of experimental colitis. Clin. Dev. Immunol. 2011:807483. doi: $10.1155 / 2011 / 807483$

Ott, S. J., Musfeldt, M., Wenderoth, D. F., Hampe, J., Brant, O., Folsch, U. R., et al. (2004). Reduction in diversity of the colonic mucosa associated bacterial microflora in patients with active inflammatory bowel disease. Gut 53, 685-693. doi: 10.1136/gut.2003.025403

Oz, H. S., Chen, T., and de Villiers, W. J. (2013). Green tea polyphenols and sulfasalazine have parallel anti-inflammatory properties in colitis models. Front. Immunol. 4:132. doi: 10.3389/fimmu.2013.00132

Sanchez-Fidalgo, S., Cardeno, A., Sanchez-Hidalgo, M., Aparicio-Soto, M., and de la Lastra, C. A. (2013). Dietary extra virgin olive oil polyphenols supplementation modulates DSS-induced chronic colitis in mice. J. Nutr. Biochem. 24, 1401-1413. doi: 10.1016/j.jnutbio.2012.11.008

Santino, A., Scarano, A., De Santis, S., De Benedictis, M., Giovinazzo, G., and Chieppa, M. (2017). Gut microbiota modulation and anti-inflammatory properties of dietary polyphenols in ibd: new and consolidated perspectives. Curr. Pharm. Des 23, 2344-2351. doi: 10.2174/1381612823666170207145420

Seregin, S. S., Golovchenko, N., Schaf, B., Chen, J., Pudlo, N. A., Mitchell, J., et al. (2017). NLRP6 protects Il10(-/-) mice from colitis by limiting colonization of Akkermansia muciniphila. Cell Rep. 19:2174. doi: 10.1016/j.celrep.2017. 05.074

Sgambato, D., Miranda, A., Ranaldo, R., Federico, A., and Romano, M. (2017). The role of stress in inflammatory bowel diseases. Curr. Pharm. Des 23, 3997-4002. doi: 10.2174/1381612823666170228123357

Shimizu, M. (2017). Multifunctions of dietary polyphenols in the regulation of intestinal inflammation. J. Food Drug Anal. 25, 93-99. doi: 10.1016/j.jfda.2016. 12.003

Shimoyama, A. T., Santin, J. R., Machado, I. D., de Oliveira e Silva, A. M., de Melo, I. L., Mancini-Filho, J., et al. (2013). Antiulcerogenic activity of chlorogenic acid in different models of gastric ulcer. Naunyn-Schmiedeberg's Arch. Pharmacol. 386, 5-14. doi: 10.1007/s00210-012-0807-2

Shin, H. S., Satsu, H., Bae, M. J., Zhao, Z., Ogiwara, H., Totsuka, M., et al. (2015). Anti-inflammatory effect of chlorogenic acid on the IL-8 production in Caco2 cells and the dextran sulphate sodium-induced colitis symptoms in C57BL/6 mice. Food Chem. 168, 167-175. doi: 10.1016/j.foodchem.2014.06.100

Siegmund, B. (2002). Interleukin-1beta converting enzyme (caspase-1) in intestinal inflammation. Biochem. Pharmacol. 64, 1-8. doi: 10.1016/S0006-2952(02) 01064-X

Sokol, H., Lay, C., Seksik, P., and Tannock, G. W. (2008). Analysis of bacterial bowel communities of IBD patients: what has it revealed? Inflamm. Bowel Dis. 14, 858-867. doi: 10.1002/ibd.20392

Sokol, H., Seksik, P., Rigottier-Gois, L., Lay, C., Lepage, P., Podglajen, I., et al. (2006). Specificities of the fecal microbiota in inflammatory bowel disease. Inflamm. Bowel Dis. 12, 106-111. doi: 10.1097/01.MIB.0000200323.38139.c6

Strober, W., and Fuss, I. J. (2011). Proinflammatory cytokines in the pathogenesis of inflammatory bowel diseases. Gastroenterology 140, 1756-1767. doi: 10.1053/ j.gastro.2011.02.016

Tegtmeyer, D., Seidl, M., Gerner, P., Baumann, U., and Klemann, C. (2017). Inflammatory bowel disease caused by primary immunodeficiencies-Clinical presentations, review of literature, and proposal of a rational diagnostic algorithm. Pediatr. Allergy Immunol. 28, 412-429. doi: 10.1111/pai.12734

Tibble, J. A., Sigthorsson, G., Bridger, S., Fagerhol, M. K., and Bjarnason, I. (2000). Surrogate markers of intestinal inflammation are predictive of relapse in patients with inflammatory bowel disease. Gastroenterology 119, 15-22. doi: 10.1053 /gast.2000.8523

Tomas-Barberan, F., Garcia-Villalba, R., Quartieri, A., Raimondi, S., Amaretti, A., Leonardi, A., et al. (2014). In vitro transformation of chlorogenic acid by human gut microbiota. Mol. Nutr. Food Res. 58, 1122-1131. doi: 10.1002/mnfr. 201300441

Tsang, M. S., Jiao, D., Chan, B. C., Hon, K. L., Leung, P. C., Lau, C. B., et al. (2016). Anti-inflammatory activities of pentaherbs formula, berberine, gallic acid and chlorogenic acid in atopic dermatitis-like skin inflammation. Molecules 21:519. doi: 10.3390/molecules21040519

Umesalma, S., and Sudhandiran, G. (2010). Differential inhibitory effects of the polyphenol ellagic acid on inflammatory mediators NF-kappaB, iNOS, COX-2, TNF-alpha, and IL-6 in 1,2-dimethylhydrazine-induced rat colon carcinogenesis. Basic Clin. Pharmacol. Toxicol. 107, 650-655. doi: 10.1111/j. 1742-7843.2010.00565.x 
Weichselbaum, L., and Klein, O. D. (2018). The intestinal epithelial response to damage. Sci. China Life Sci. 61, 1205-1211. doi: 10.1007/s11427-0189331-y

Wu, X., and Tian, Z. (2017). Gut-liver axis: gut microbiota in shaping hepatic innate immunity. Sci. China Life Sci. 60, 1191-1196. doi: 10.1007/s11427-0179128-3

Yin, J., Li, Y., Han, H., Chen, S., Gao, J., Liu, G., et al. (2018). Melatonin reprogramming of gut microbiota improves lipid dysmetabolism in high-fat diet-fed mice. J. Pineal Res. 65:e12524. doi: 10.1111/jpi.12524

Zatorski, H., Salaga, M., Zielinska, M., Piechota-Polanczyk, A., Owczarek, K., Kordek, R., et al. (2015). Experimental colitis in mice is attenuated by topical administration of chlorogenic acid. Naunyn-Schmiedeberg's Arch. Pharmacol. 388, 643-651. doi: 10.1007/s00210-015-1110-9

Zhang, H., DiBaise, J. K., Zuccolo, A., Kudrna, D., Braidotti, M., Yu, Y., et al. (2009). Human gut microbiota in obesity and after gastric bypass. Proc. Natl. Acad. Sci. U.S.A. 106, 2365-2370. doi: 10.1073/pnas.0812600106

Zhang, J., Dou, W., Zhang, E., Sun, A., Ding, L., Wei, X., et al. (2014). Paeoniflorin abrogates DSS-induced colitis via a TLR4-dependent pathway. Am. J. Physiol. Gastrointest. Liver Physiol. 306, G27-G36. doi: 10.1152/ajpgi.00465.2012

Zhang, X., Huang, H., Yang, T., Ye, Y., Shan, J., Yin, Z., et al. (2010). Chlorogenic acid protects mice against lipopolysaccharide-induced acute lung injury. Injury 41, 746-752. doi: 10.1016/j.injury.2010.02.029
Zhang, Y., Dong, A., Xie, K., and Yu, Y. (2018). Dietary supplementation with high fiber alleviates oxidative stress and inflammatory responses caused by severe sepsis in mice without altering microbiome diversity. Front. Physiol. 9:1929. doi: 10.3389/fphys.2018.01929

Zhao, Z., Shin, H. S., Satsu, H., Totsuka, M., and Shimizu, M. (2008). 5caffeoylquinic acid and caffeic acid down-regulate the oxidative stress- and TNF-alpha-induced secretion of interleukin-8 from Caco- 2 cells. J. Agric. Food Chem. 56, 3863-3868. doi: 10.1021/jf073168d

Zheng, Y., Liu, J., Cao, M. L., Deng, J. M., and Kou, J. (2016). Extrication process of chlorogenic acid in Crofton weed and antibacterial mechanism of chlorogenic acid on Escherichia coli. J. Environ. Biol. 37, 1049-1055.

Conflict of Interest Statement: The authors declare that the research was conducted in the absence of any commercial or financial relationships that could be construed as a potential conflict of interest.

Copyright (c) 2019 Zhang, Jiao, Wang, Lin and You. This is an open-access article distributed under the terms of the Creative Commons Attribution License (CC BY). The use, distribution or reproduction in other forums is permitted, provided the original author(s) and the copyright owner(s) are credited and that the original publication in this journal is cited, in accordance with accepted academic practice. No use, distribution or reproduction is permitted which does not comply with these terms. 